“C 2018 IEEE. Personal use of this material is permitted. Permission from IEEE must be obtained for all other uses, in any current or future media, including reprinting/republishing this material for advertising or promotional purposes, creating new collective works, for resale or redistribution to servers or lists, or reuse of any copyrighted component of this work in other works." 


\title{
Metamaterial-inspired Compact Directive Antenna Systems
}

\author{
$\underline{\text { Richard W. Ziolkowski }}$ \\ University of Technology Sydney, Global Big Data Technologies Centre, \\ Ultimo NSW 2000, Australia \\ Richard.Ziolkowski@uts.edu.au
}

\begin{abstract}
Huygens dipole and multipole antennas are briefly reviewed. These electrically small systems provide enhanced directivity, a feature highly desired for current and future wireless platforms. The design, simulation, fabrication, and measurement results for the Huygens dipole antennas are discussed. The Huygens multipole antenna concepts are described. These brief examinations will be greatly expanded upon during my presentation.
\end{abstract}

\section{INTRODUCTION}

Electrically-small antennas (ESAs) have drawn much recent attention. Their compact structures are preferred for wireless devices. Much progress has been made for practical applications, including RFIDs [1], wireless biomedical systems [2], and wireless power transfer (WPT) [3]. Nevertheless, the demand for ESAs that attain outstanding performance characteristics continues unabated.

One area of practical interest, particularly for the next generation (5G) of wireless systems, is highly directive antennas. Unlike most ESAs which radiate dipole-like radiation patterns with low gain and front-to-back ratios (FTBRs), Huygens dipole ESAs have been demonstrated that are compact, low-profile, and able to generate broadside radiation patterns with good FTBRs, wide beamwidths, linear or circular polarization, high radiation efficiencies, and large bandwidths. Many of these desirable performance characteristics have been demonstrated [4]-[7]. It has been demonstrated recently that needle-like radiation is possible from electrically small systems with combinations of Huygens multipole elements [8]. This combination of higher order modes (HOMs) achieves high directivity with low sidelobes and large front-to-back ratios (FTBRs). Most recently, it has been demonstrated that multi-layered electrically small structures can simultaneously support these HOMs to achieve high directivities [9]. Progress in highly directive electrically small platforms will be reviewed in the presentation.

\section{HUYGENS DiPOLE ANTENNAS}

An electrically-small Huygens circularly-polarized near-field resonant parasitic (NFRP) antenna and its performance characteristics were presented in [7]. They were verified with measured results. Circularly polarized performance was attained by integrating the NFRP electric (Egyptian axe dipole, EAD) and magnetic (capacitively loaded loop, CLL) elements as shown in Fig. 1a. The fabricated prototype is shown in Fig. 1b. The measured cardioid pattern in the principal left-hand CP (LHCP) state is compared to the non-excited right-hand $\mathrm{CP}$ (RHCP) state in Fig. 1c.

To realize its unique CP cardioid-shaped radiation characteristics in a compact structure, the illustrated crossed-dipole configuration was employed. The EAD (CLL) elements act as the orthogonal electric dipole (magnetic dipole) radiators. Balanced broadside-radiated electric and magnetic field amplitudes with the requisite $90^{\circ}$ phase difference between them are realized by exciting these two pairs of electric and magnetic dipoles with a specially designed, unbalanced crossed-dipole structure. The electrically small $(\mathrm{ka}=0.73)$ prototype operated at $1584 \mathrm{MHz}$ with a $0.6 \mathrm{~dB}$ axial ratio and was low profile, $0.04 \lambda_{0}$. The measured HCP peak realized LHCP gain was $2.7 \mathrm{dBic}$ and the associated front-to-back ratio was $17.7 \mathrm{~dB}$. 

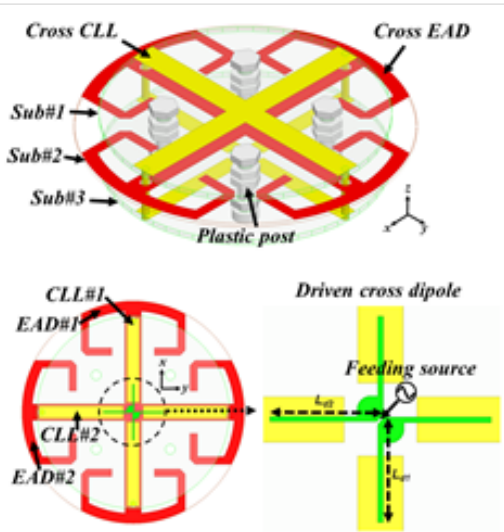

HCP Antenna Model

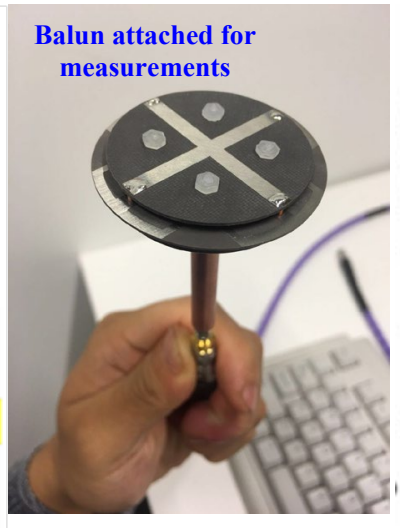

HCP Prototype

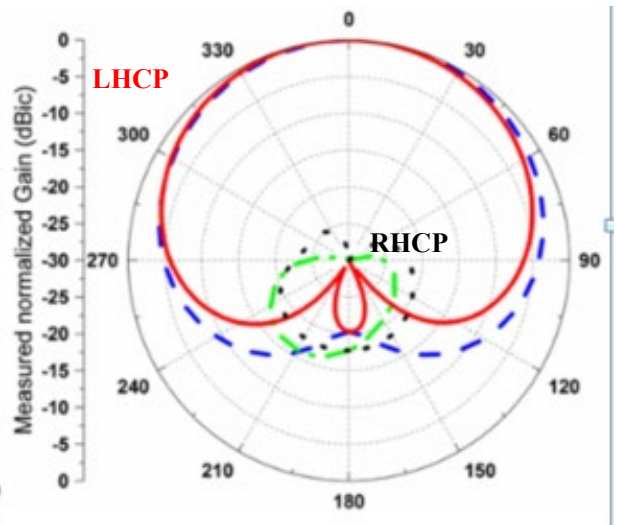

Measured Realized Gain Pattern

Fig. 1. Huygens circular polarized antenna. (a) HFSS model. (b) Fabricated prototype. (c) Measured realized gain patterns of both the excited left (LHCP) and unexcited right (RHCP) hand polarization states.

\section{High DiRectivity With Higher ORDER HuYGENS MULTIPOLES}

For nearly a century, the concept of "needle radiation" has captured the attention of the electromagnetics communities in both physics and engineering for nearly a century [10]. While it is well-known that superdirective solutions exist and can suffer ill-posedness issues in principle, the concept of combining Huygens higher order multipoles suggests that needle-like directivity can be obtained even with an electrically small platform [8]. The directivity of a system employing $N$ Huygens higher order multipoles is

$$
D(\theta, \varphi)=\frac{2(1+\cos \theta)^{2} P_{N}^{2}(\theta)}{\int_{0}^{\pi} d \theta \sin \theta(1+\cos \theta)^{2} P_{N}^{2}(\theta)}
$$

The outcome for $N=1$ (dipole only), 2 (dipole and quadrupole), and 5 (dipole to pentapole) is shown in Fig. 2 , both in a linear and in a $\mathrm{dB}$ scale. The increasingly directive result is clear in Fig. 2a and the lack of sidelobes and large FTBR outcome is highlighted with Fig. 2 b.
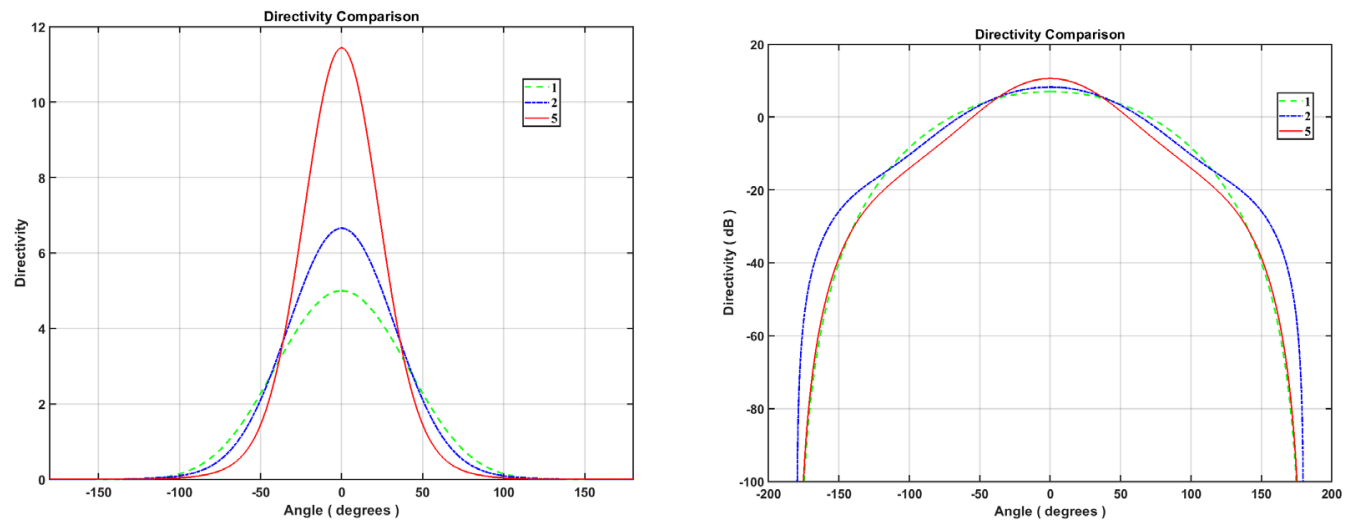

Fig. 2. Directivity patterns attained with $N$ Huygens multipoles. (a) Linear scale. (b) dB scale.

An example to illustrate how such a HHOM configuration could be achieved in shown in Fig. 3. As Fig. 3a shows, an electric dipole and a loop antenna are combined to form the electrically small (at $300 \mathrm{MHz}$ ) Huygens 
dipole pair (center) and two Huygens quadrupole antennas (outside pairs). Each quadrupole system is achieved with two pairs of Huygens dipole pairs excited with opposite current directions. From the 3D directivity pattern in Fig. 3b, the peak directivity is 6.3, which nearly recovers the ideal value shown in Fig. 2, 6.6. The symmetry of the directivity, the lack of sidelobes, and the large FTBR value $(24.4 \mathrm{~dB})$ are clear in Figs. $6.3 \mathrm{~b}$ and $6.3 \mathrm{c}$.
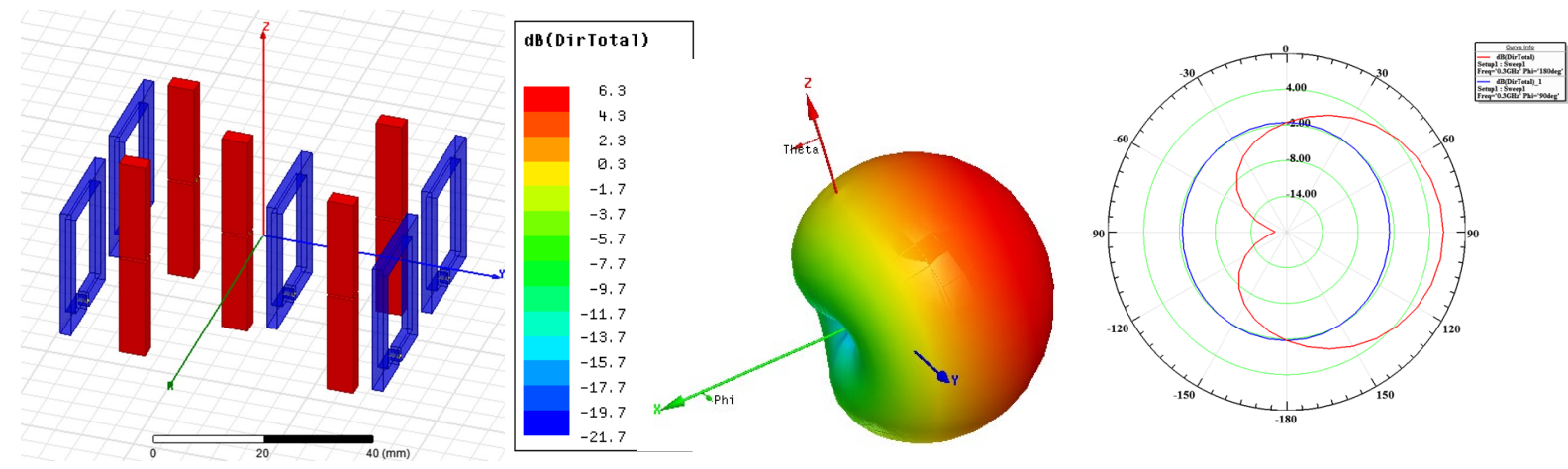

Fig. 3. Huygens $\mathrm{N}=2$ multipole system. (a) HFSS model. (b) 3D directivity pattern. (c) 2D directivity patterns.

\section{CONCLUSION}

Several features of realized Huygens dipole antennas were described. They will be emphasized in the presentation. The Huygens higher order multipole systems were reviewed. An example of an approach to realize such an antenna system was given. The multi-layered metamaterial-inspired structure [9] will be highlighted in the presentation.

\section{REFERENCES}

[1] J. Zhang and Y. Long, "A novel metal-mountable electrically small antenna for RFID tag applications with practical guidelines for the antenna design," IEEE Trans. Antennas Propag., vol. 62, no. 11, $\quad$ pp. 5820-5829, Nov. 2014.

[2] H. Wong, W. Lin, L. Huitema and E. Arnaud, "Multi-polarization reconfigurable antenna for wireless biomedical applications," IEEE Trans. Biomed. Circuits Syst., vol. 11, no. 3, pp. 652-660, Jun. 2017.

[3] I.-J. Yoon and H. Ling, "Realizing efficient wireless power transfer using small folded cylindrical helix dipoles," IEEE Antennas Wireless Propag. Lett., vol. 9, pp. 846-849, 2010.

[4] P. Jin and R. W. Ziolkowski, "Metamaterial-inspired, electrically small Huygens sources," IEEE Antennas Wirel. Propag. Lett., vol.9, pp. 501-505, 2010.

[5] M. C. Tang, H. Wang and R. W. Ziolkowski, "Design and testing of simple, electrically small, low-profile, Huygens source antennas with broadside radiation performance," IEEE Trans. Antennas Propag., vol. 64, no. 11, pp. 4607-4617, Nov. 2016.

[6] M.-C. Tang, T. Shi, and R. W. Ziolkowski, "Electrically small, broadside radiating Huygens source antenna augmented with internal non-Foster elements to increase its bandwidth," IEEE Antennas Wirel. Propag. Lett., vol. 16, pp. 712-715, 2017.

[7] W. Lin and R. W. Ziolkowski, "Electrically-small, low-profile, Huygens circularly polarized antenna," IEEE Trans. Antennas Propag., vol. 66, no. 2, pp. 636-643, Feb. 2018.

[8] R. W. Ziolkowski, "Using Huygens multipole arrays to realize unidirectional needle-like radiation," Phys. Rev. X, vol. 7, $031017,2017$.

[9] S. Arslanagić and R. W. Ziolkowski, "Highly sub-wavelength, superdirective cylindrical nanoantennas," Phys. Rev. Lett., Under Review, Feb. 2018.

[10] C. W. Oseen, Die einsteinsche nadelstichstrahlung und die maxwellschen gleichungen, Annalen der Physik, vol. 374, no. 19 , pp. 202--204, 1922. 